\title{
Dendritic Growth Morphologies in Al-Zn Alloys-Part II: Phase-Field Computations
}

\begin{abstract}
J.A. DANTZIG, PAOLO DI NAPOLI, J. FRIEDLI, and M. RAPPAZ
In Part I of this article, the role of the $\mathrm{Zn}$ content in the development of solidification microstructures in Al-Zn alloys was investigated experimentally using X-ray tomographic microscopy. The transition region between $\langle 100\rangle$ dendrites found at low $\mathrm{Zn}$ content and $\langle 110\rangle$ dendrites found at high $\mathrm{Zn}$ content was characterized by textured seaweed-type structures. This Dendrite Orientation Transition (DOT) was explained by the effect of the $\mathrm{Zn}$ content on the weak anisotropy of the solid-liquid interfacial energy of Al. In order to further support this interpretation and to elucidate the growth mechanisms of the complex structures that form in the DOT region, a detailed phase-field study exploring anisotropy parameters' space is presented in this paper. For equiaxed growth, our results essentially recapitulate those of Haxhimali et al. ${ }^{[1]}$ in simulations for pure materials. We find distinct regions of the parameter space associated with $\langle 100\rangle$ and $\langle 110\rangle$ dendrites, separated by a region where hyperbranched dendrites are observed. In simulations of directional solidification, we find similar behavior at the extrema, but in this case, the anisotropy parameters corresponding to the hyperbranched region produce textured seaweeds. As noted in the experimental work reported in Part I, these structures are actually dendrites that prefer to grow misaligned with respect to the thermal gradient direction. We also show that in this region, the dendrites grow with a blunted tip that oscillates and splits, resulting in an oriented trunk that continuously emits side branches in other directions. We conclude by making a correlation between the alloy composition and surface energy anisotropy parameters.
\end{abstract}

DOI: $10.1007 / \mathrm{s} 11661-013-1911-8$

(C) The Minerals, Metals \& Materials Society and ASM International 2013

\section{INTRODUCTION}

IN a companion article, ${ }^{[2]} \mathrm{X}$-ray tomographic microscopy was used to characterize the microstructures that form in directionally solidified (DS) and quenched $\mathrm{Al}-\mathrm{Zn}$ alloys for various $\mathrm{Zn}$ compositions ranging from 5 to $90 \mathrm{wt}$ pct. The results obtained in that study were in agreement with those previously found by Gonzales and Rappaz $^{[3,4]}$ on DS (but not quenched) Al-Zn alloys. In summary, for alloys containing less than 25 wt pet Zn, $\langle 100\rangle$ dendrites were observed, and for alloys containing more than $55 \mathrm{wt}$ pct $\mathrm{Zn},\langle 110\rangle$ dendrites were found. In the intermediate region between these two bounding compositions, textured seaweed structures appear. This

J.A. DANTZIG, Professor, is with the Laboratoire de Simulation des Matériaux, Ecole Polytechnique Fédérale de Lausanne, EPFLSTI-IMX-LSMX, Station 12, 1015 Lausanne, Switzerland, and also with the Department of Mechanical Science and Engineering, University of Illinois, 1206 West Green Street, Urbana, IL 61801. PAOLO DI NAPOLI, Postdoctoral Researcher, and M. RAPPAZ, Professor, are with the Laboratoire de Simulation des Matériaux, Ecole Polytechnique Fédérale de Lausanne, EPFL-STI-IMX-LSMX. Contact e-mail: paolo.dinapoli@epfl.ch J. FRIEDLI, formerly Ph.D. Student with the Laboratoire de Simulation des Matériaux, Ecole Polytechnique Fédérale de Lausanne, EPFL-STI-IMX-LSMX, is now Development Engineer with the Novelis Inc., Sierre, Switzerland.

Manuscript submitted April 2, 2013. transition from $\langle 100\rangle$ to $\langle 110\rangle$ was called the "dendrite orientation transition" (DOT).

Haxhimali et al. ${ }^{[1]}$ and Gonzales and Rappaz ${ }^{[3,4]}$ attributed the DOT to a modification of the weak anisotropy of the solid-liquid interfacial energy $\gamma_{s \ell}$ of Al by the addition of $\mathrm{Zn}$, an element with very high interfacial energy anisotropy in its pure state. Haxhimali performed phase-field simulations in which the anisotropy parameters were systematically varied to explore the role of these parameters on the resulting microstructures, but only for pure materials solidifying as equiaxed crystals in an undercooled melt. Our objective in this work is to use phase-field simulations for binary alloys to further explore the role of the interfacial energy anisotropy on the selection of dendrite orientation. We consider first equiaxed growth, where we find results quite similar to those of Haxhimali et al., and then proceed to examine directional solidification (DS), where we find that the additional constraint of an imposed temperature gradient helps to discriminate the effect of changes in $\gamma_{s e}$. Throughout this article, we assume that interface attachment kinetics can be neglected.

This paper is organized as follows: We first describe the various representations for the anisotropy in $\gamma_{s \ell}$ found in the literature and develop some guiding "principles" for the selection of dendrite growth directions. We then describe the implementation of the anisotropy in an adaptive finite element-based phasefield code and present results of a systematic exploration 
of anisotropy parameter space. Finally, we interpret those results in terms of the DOT principles and relate the observations to the composition of Al- $\mathrm{Zn}$ alloys.

\section{INTERFACIAL ENERGY AND ITS ANISOTROPY}

Several functional forms have been used in the literature to represent the anisotropy of $\gamma_{s \ell}$ for systems having cubic symmetry. We show that some of these representations are incompatible, meaning that one cannot always convert directly from one form into another. This is important to recognize when comparing computations performed using different representations.

To examine the morphological stability of an initially spherical solid particle, Mullins and Sekerka ${ }^{[5]}$ introduced small surface perturbations given in terms of spherical harmonics $Y_{l}^{m}(\theta, \varphi)$, with $\theta \in[0, \pi]$ and $\varphi \in[0,2 \pi]$ being the polar and azimuthal coordinates, respectively. Chan et al., ${ }^{[6]}$ using considerations based on group theory, proposed that one should consider a restricted set of the $Y_{l}^{m}$-consistent symmetry of the underlying crystal structure. To that end, they introduced the so-called cubic harmonics, a symmetry-adapted orthogonal basis with cubic symmetry. The combinations of $l$ and $m$ respecting this symmetry are thus restricted to $l=4,6,8,10, \ldots$ and $m=0, \pm 4, \pm 8, \pm 12, \ldots$ with $|m| \leq l$.

The lowest order cubic harmonics are

$$
\begin{aligned}
\mathcal{K}_{0} & =1 \\
\mathcal{K}_{4}(\theta, \varphi) & =N_{4}\left[Y_{4}^{0}+\sqrt{5 / 14}\left(Y_{4}^{4}+Y_{4}^{-4}\right)\right] \\
\mathcal{K}_{6}(\theta, \varphi) & =N_{6}\left[Y_{6}^{0}-\sqrt{7 / 2}\left(Y_{6}^{4}+Y_{6}^{-4}\right)\right]
\end{aligned}
$$

The interfacial energy is then written as a summation over this basis

$$
\gamma_{s \ell}(\theta, \varphi)=\gamma_{s \ell}^{0}\left[1+\delta_{4} \mathcal{K}_{4}+\delta_{6} \mathcal{K}_{6}+\cdots\right]
$$

The $N_{i}$ in Eqs. [1] are normalization constants, which Chan et al. ${ }^{[6]}$ chose such that each $\mathcal{K}_{i}$ has unit amplitude in the polar direction in spherical coordinates. The choice of coefficients is not unique. For example, one may choose the $N_{i}$ instead such that the mean amplitude integrated over the unit sphere is one. ${ }^{[7]}$

Fehlner and Vosko ${ }^{[7]}$ proposed an alternative basis to that given in Eq. [1] that is more convenient for computations. They defined two functions $Q$ and $S$ respecting the fourfold symmetry of cubic crystals:

$$
\begin{gathered}
Q=n_{x}^{4}+n_{y}^{4}+n_{z}^{4}=\sin ^{4} \theta\left(\cos ^{4} \varphi+\sin ^{4} \varphi\right)+\cos ^{4} \theta \\
S=n_{x}^{2} n_{y}^{2} n_{z}^{2}=\sin ^{4} \theta \cos ^{2} \theta \sin ^{2} \varphi \cos ^{2} \varphi
\end{gathered}
$$

where the $n_{i}$ are the Cartesian components of the unit normal vector $\vec{n}$. The interfacial energy is then written as

$\gamma_{s \ell}(\vec{n})=\gamma_{s \ell}^{0}\left[1+a_{1}\left(Q-\frac{3}{5}\right)+a_{2}\left(3 Q+66 S-\frac{17}{7}\right)+\cdots\right]$
The formulations given in Eqs. [2] and [5] are equivalent, ${ }^{[8]}$ with $\delta_{4}=(4 \sqrt{\pi} / 15) a_{1}$ and $\delta_{6}=(8 \sqrt{\pi} /(7 \sqrt{13})) a_{2}$.

Another description for the anisotropy widely used in phase-field simulations of dendritic growth in cubic materials is given by (cf. $\left.{ }^{[9]}\right)$

$$
\gamma_{s \ell}(\vec{n})=\gamma_{s \ell}^{0}\left(1-3 \varepsilon_{4}\right)\left[1-\frac{4 \varepsilon_{4}}{1-3 \varepsilon_{4}} Q\right]=\gamma_{s \ell}^{0}\left[1+\varepsilon_{4}(4 Q-3)\right]
$$

In the plane $z=0$, Eq. [6] reduces to the simple form $\gamma_{s \ell}=\gamma_{s \ell}^{0}\left[1+\varepsilon_{4} \cos 4 \varphi\right]$, and in the planes $x=0$ and $y=0$, one obtains the equivalent form $\gamma_{s \ell}=\gamma_{s \ell}^{0}\left[1+\varepsilon_{4} \cos 4 \theta\right]$. It is important to note that $\varepsilon_{4}$ cannot be written in terms of the $a_{i}$ 's in Eq. [5]. The closest possible form can be obtained by setting $a_{1}=4 \varepsilon_{4}$ and $a_{2}=0$, but then the isotropic parts of the surface energy do not match in the two formulations. As this result makes clear, one must be very careful when comparing results of calculations and experiments performed using different representations for the anisotropy.

When $\varepsilon_{4}$ is positive, $\gamma_{s \ell}$ in Eq. [6] always produces $\langle 100\rangle$ dendrites, whereas $\varepsilon_{4}<0$ always results in $\langle 111\rangle$ dendrites. Thus, no $\langle 110\rangle$ dendrites can be generated from the representation given by Eq. [6]. On the other hand, the expression given in Eq. [5] produces $\langle 100\rangle$ dendrites for $a_{1}>0$ and small $a_{2}$, or $\langle 110\rangle$ dendrites for $a_{2}<0$ and small $a_{1}$. For $a_{1}<0$ and small $a_{2},\langle 111\rangle$ dendrites appear, and for $a_{2}<0$ and small $a_{1}$, we obtain mixed $\langle 100\rangle$ and $\langle 111\rangle$ dendrites. We will show that one can indeed produce a continuous transition between $\langle 100\rangle$ and $\langle 110\rangle$ in the quadrant of parameter space defined by $\left(a_{1}>0, a_{2}<0\right)$.

\section{A. Simplified Criteria for DOT}

It would be convenient if one were able to define a criterion, expressed in the form of some combination of the anisotropy parameters $\left(a_{1}, a_{2}\right)$, that would determine a particular dendrite growth orientation. The problem will turn out to be too complicated for this to be the case, but there are two simple criteria that have been proposed to understand the structures that appear in the numerical calculations.

The simplest criterion to select dendrite trunk (and side branch) growth directions assumes that they develop along the directions of the highest surface energy, so as to minimize the corresponding surfaces. In simple cases, the highest surface energy directions correspond to minima of what is known as the solidliquid interface stiffness. First introduced by Herring, ${ }^{[10]}$ and later extended by Du et al., ${ }^{[1]}$ the interface stiffness $\boldsymbol{\Phi}$ is a symmetric tensor that incorporates the anisotropy of the surface energy, defined as

$$
\Phi_{i j}=\gamma_{s \ell} \delta_{i j}+\frac{\partial^{2} \gamma_{s \ell}}{\partial \xi_{i} \partial \xi_{j}}
$$

where $\delta_{i j}$ is the Kronecker delta and $\xi_{1}$ and $\xi_{2}$ define coordinates along orthogonal directions on the surface perpendicular to the unit normal vector $\vec{n}$. We will characterize $\boldsymbol{\Phi}$ by its trace, which is invariant under 
rotation around $\vec{n}$. In spherical coordinates, where $\boldsymbol{\xi}_{1}=\hat{\boldsymbol{\theta}}$ and $\boldsymbol{\xi}_{2}=\hat{\boldsymbol{\varphi}}$, we have

$$
\operatorname{tr}(\Phi)=2 \gamma_{s \ell}+\frac{1}{\sin \theta} \frac{\partial}{\partial \theta}\left(\sin \theta \frac{\partial \gamma_{s \ell}}{\partial \theta}\right)+\frac{1}{\sin ^{2} \theta} \frac{\partial^{2} \gamma_{s \ell}}{\partial \varphi^{2}}
$$

Herring ${ }^{[10]}$ proposed that for a crystal in equilibrium with its liquid, the shape will be such that the chemical potential is constant at all points on the solid-liquid interface. Assuming the diffusion of heat to be much faster than solute diffusion, i.e., the interface is isothermal, Herring derived an expression for the liquid composition $C_{\ell}^{0}$ at the solid-liquid interface of an equilibrium shape crystal, given by

$$
C_{\ell}^{0}=C_{\infty}+\frac{1}{m_{\ell} \Delta S_{\mathrm{f}}}\left[\left(\gamma_{s \ell}+\frac{\partial^{2} \gamma_{s \ell}}{\partial \xi_{1}^{2}}\right) K_{1}+\left(\gamma_{s \ell}+\frac{\partial^{2} \gamma_{s \ell}}{\partial \xi_{2}^{2}}\right) K_{2}\right]
$$

where $C_{\infty}$ is the nominal melt composition, $m_{\ell}$ the slope of the liquidus $(<0), \Delta S_{\mathrm{f}}$ the volumetric entropy of fusion, and $K_{1}$ and $K_{2}$ are the principal curvatures, i.e., the eigenvalues of the curvature tensor $\boldsymbol{\kappa}$. Equation [9] was expressed in a more general form by $\mathrm{Du}$ et al..$^{11]}$

$$
C_{\ell}^{0}=C_{\infty}+\frac{1}{m_{\ell} \Delta S_{\mathrm{f}}} \boldsymbol{\Phi}: \boldsymbol{\kappa}
$$

If the surface energy is isotropic, $\gamma_{s \ell}(\vec{n})=\gamma_{s \ell}^{0}$, and Eq. [9] reduces to the well-known Gibbs-Thomson equation.

For the particular case of a sphere, where $K_{1}=K_{2}=1 / R$, the square brackets in Eq. [9] reduce to $[\operatorname{tr}(\boldsymbol{\Phi}) / R]$. Figure 1(a) shows the ratio $\gamma_{s \ell}(\vec{n}) / \gamma_{s \ell}^{0}$ computed using Eq. [5] for $\vec{n}=\langle 100\rangle$ and $\vec{n}=\langle 110\rangle$, on the surface of a sphere in the quadrant of parameter space $\left(a_{1} \geq 0, a_{2} \leq 0\right)$. As can be seen, the surface energy has two distinct regions. For small values of $a_{1}$, the $\langle 110\rangle$ directions have higher surface energy than the $\langle 100\rangle$, whereas at higher values of $a_{1}$, the situation is reversed. The two regions are separated by a line given by $a_{1}=-3 a_{2}$.

Examination of Eq. [5] shows that the coefficient multiplying $Q$ changes sign when crossing this line. Thus, one might expect to see $\langle 110\rangle$ dendrites for parameter sets below this line, and $\langle 100\rangle$ dendrites above it. This is our first criterion. To examine the second principle that the crystal grows most easily in the direction where the surface stiffness is minimum, i.e., the direction where the surface can be deformed most easily, we plot $\gamma_{s e}^{0} / \operatorname{tr}(\Phi)$ in Figure 1(b), for the same directions $\vec{n}=\langle 100\rangle$ and $\langle 110\rangle$. Once again, the quadrant of interest in anisotropy parameter space is divided into two regions by a line, this time given by $a_{1}=-20 a_{2} / 3$, above which the minimum stiffness is achieved in the $\langle 100\rangle$ direction, and below which the minimum stiffness corresponds to the $\langle 110\rangle$ direction. On the boundary separating these two regions, there is no preferred direction, and we can anticipate that seaweed structures will appear in the neighborhood of this boundary.



(a) Surface energy $\gamma_{s \ell} / \gamma_{s \ell}^{0}$



(b) Inverse stiffness $\gamma_{s \ell}^{0} / \operatorname{tr}(\Phi)$

Fig. 1-Surface energy and reciprocal of $\operatorname{tr}(\Phi)$ for a sphere in the quadrant of interest in parameter space for the anisotropy function given by Eq. [5], for $\vec{n}=\langle 100\rangle$ and $\langle 110\rangle$.

Considering the second principle, which has a sounder physical basis, a sharp transition between $\langle 100\rangle$ and $\langle 110\rangle$ should occur at $a_{1}=-20 / 3 a_{2}$, a value for which $\operatorname{tr}(\Phi)$ is degenerate in a (001) plane. However, as shown by Friedli, ${ }^{[12]}$ the equilibrium shape of crystals, calculated with the help of the $\xi$-vector formalism of Cahn and Hoffman, ${ }^{[13]}$ shows a more complex behavior and does not exactly follow that based on the minimum stiffness trace. Firstly, it is only at small values of $a_{1}$ that both principal curvatures of the equilibrium crystal maximize at $\langle 110\rangle$, and at small values of $-a_{2}$ that both principal curvatures maximize at $\langle 100\rangle$. For intermediate values of $\left(a_{1},-a_{2}\right)$, one of the two principal curvatures of the equilibrium crystal is maximum for $\langle 100\rangle$, while the other is maximum for $\langle 110\rangle$. Secondly, the transition between $\langle 100\rangle$ and $\langle 110\rangle$ predicted from the maximum curvature is not a linear function of $a_{2}$ and deviates from the line predicted by the minimum stiffness trace when $\left(-a_{2}\right)$ increases. For example, when $a_{2}=-0.01$, the minimum stiffness trace criterion predicts an abrupt change of dendrite growth direction for $a_{1}=0.066$, whereas a criterion based on the maximum mean curvature of the $\xi$-vector predicts a transition for $a_{1}=0.11$.

This shows clearly that a refined criterion for dendrite orientation selection is needed in such complex situations, 
when the two principal curvatures do not maximize at a single orientation. In order to explore anisotropy parameter space more precisely, we simulate the dendritic growth process using the phase-field method, which we develop in the next section.

\section{PHASE-FIELD MODEL}

We simulate dendritic growth using the phase-field formulation for binary alloys first introduced by $\mathrm{Karma}^{[14]}$ and later extended by Echebarria et al. ${ }^{[15]}$ The model is described here only to a depth sufficient to understand and interpret the results, and the reader is referred to the original works for further details.

The phase-field $\psi \in[-1,1]$ is defined such that $\psi=+1$ corresponds to the solid, $\psi=-1$ to the liquid, and intermediate values are associated with the diffuse solid-liquid interface. The solid-liquid interface thus has a finite width, given in the model by $W_{0} \eta(\vec{n})$, where $\eta(\vec{n})$ embodies the anisotropy. The time scale for evolution of the phase field is $\tau_{0} \eta^{2}(\vec{n})$.

The evolution equation for $\psi$, with length scaled by $W_{0}$ and time by $\tau_{0}$, is given by

$$
\begin{aligned}
& {\left[1-\left(1-k_{0}\right) \tilde{\Theta}\right] \eta^{2}(\vec{n}) \frac{D \psi}{D \tau}=\nabla \cdot\left[\eta^{2}(\vec{n}) \nabla \psi\right]} \\
& \quad+\sum_{i} \frac{\partial}{\partial x_{i}}\left[|\nabla \psi|^{2} \eta(\vec{n}) \frac{\partial \eta(\vec{n})}{\partial\left(\partial_{x_{i}} \psi\right)}\right] \\
& \quad+\left(1-\psi^{2}\right)\left[\psi-\frac{\lambda}{1-k_{0}}\left(1-\psi^{2}\right)(\tilde{U}+\tilde{\Theta})\right]
\end{aligned}
$$

where $k_{0}$ is the segregation coefficient, taken to be constant, and $\lambda$ is a phase-field model parameter defined below in Eq. [16]. We consider two configurations: equiaxed growth of a spherical seed in an undercooled isothermal melt; and DS, where the sample is pulled at constant velocity $v_{\mathrm{p}}$ through a "frozen" linear temperature field characterized by the temperature gradient $G$. The two configurations are distinguished in Eq. [11] by the definition of $\tilde{\Theta}$

$$
\tilde{\Theta}= \begin{cases}0 & \text { Equiaxed } \\ \tilde{x}-\tilde{x}_{0}-\left(\tilde{v}_{\mathrm{P}}-\tilde{v}_{\mathrm{F}}\right) \tau / \tilde{l}_{\mathrm{T}} & \mathrm{DS}\end{cases}
$$

The DS computations are performed in a frame moving at velocity $\tilde{v}_{\mathrm{F}}$ in the $\tilde{x}$ direction, and the notation $D / D \tau=$ $\partial / \partial \tau-\tilde{v}_{F}(\partial / \partial x)$ has the usual interpretation of the advective derivative. The offset $\tilde{x}_{0}$ is used to limit the computation to a desired region near the dendrite tip. The dimensionless position along the growth axis $\tilde{x}=x / W_{0}$ is thus reduced by $\tilde{x}_{0}+\left(\tilde{v}_{\mathrm{P}}-\tilde{v}_{\mathrm{F}}\right) \tau_{0} / W_{0}$ and scaled by the approximate length of the mushy zone $l_{\mathrm{T}}$, also called the "thermal length," $\tilde{l}_{\mathrm{T}}=l_{\mathrm{T}} / W_{0}=m_{\ell}\left(k_{0}-1\right) C_{\ell}^{0} / G W_{0}$, where $m_{\ell}$ is the slope of the liquidus line and $C_{\ell}^{0}=$ $\left(T_{0}-T_{\mathrm{f}}\right) / m_{\ell}$ is a reference concentration and $T_{0}$ is the corresponding reference temperature. For the equiaxed case, $T_{0}$ is the isothermal melt temperature. In DS, $T_{0}$ is the temperature at the base of the computational domain, discussed further in Section IV-B.
The concentration $C$ is represented in scaled form via $\tilde{U}^{[15]}$, defined as

$$
\tilde{U}=\frac{1}{1-k_{0}}\left[\frac{2 C / C_{\ell}^{0}}{1+k_{0}-\left(1-k_{0}\right) \psi}-1\right]
$$

The solute balance is given by a modified Fick's Law

$$
\begin{aligned}
\left(\frac{1+k_{0}}{2}-\frac{1-k_{0}}{2} \psi\right) \frac{D \tilde{U}}{D \tau}= & \nabla \cdot\left(\tilde{D}_{\ell} \frac{1-\psi}{2} \nabla \tilde{U}+\vec{j}\right) \\
& +\frac{1+\left(1-k_{0}\right) \tilde{U}}{2} \frac{D \psi}{D \tau}
\end{aligned}
$$

where the scaled diffusivity is $\tilde{D}_{\ell}=D_{\ell} \tau_{0} / W_{0}^{2}$. Equation [14] includes an "anti-trapping" current $\vec{j}$, introduced in order to cancel artificial trapping of solute due to the diffuse interface, ${ }^{[14]}$ given by

$$
\vec{j}=\frac{1}{2 \sqrt{2}}\left[1+\left(1-k_{0}\right) \tilde{U}\right]\left(\frac{D \psi}{D \tau} \frac{\nabla \psi}{|\nabla \psi|}\right)
$$

The term in parentheses represents the normal velocity of the interface. Note that we have not included thermal noise in our simulations.

The chemical capillary length $d_{0}=\Gamma_{s \ell} /\left(\left|m_{\ell}\right|\left(1-k_{0}\right) C_{\infty}\right)$, where $\Gamma_{s \ell}=\gamma_{s l}^{0} / \Delta S_{\mathrm{f}}$ is the Gibbs-Thomson coefficient, and the interface attachment coefficient $\beta$ are related to the phase-field parameters via

$$
d_{0}=\alpha_{1} W_{0} / \lambda ; \quad \beta=\alpha_{1} \frac{\tau}{\lambda W_{0}}\left[1-\alpha_{2} \frac{\lambda W_{0}^{2}}{\tau_{0} D}\right]
$$

where $\alpha_{1}=0.8839$ and $\alpha_{2}=0.6267$ are chosen to ensure that the phase-field model converges to the sharp interface model. See Echebarria et al. ${ }^{[15]}$ for further details. The physical parameters used in the simulations are listed in Table I. The diffusion coefficient was purposely decreased by a factor of approximately 2 to allow larger time steps to be used in the explicit solution scheme.

The length scales of the grid spacing $\left(\Delta \tilde{x} \approx W_{0}\right.$ to resolve the interface) and the simulation domain $\left(L \gg \tilde{D}_{\ell} / \tilde{v}_{\text {tip }}\right.$, where $v_{\text {tip }}$ is the speed of the dendrite tip, to avoid interaction between the solute field and the boundaries) are widely different. We therefore solve these equations on an adaptive finite element grid. ${ }^{[21-26]}$ The phase field in the interfacial region is always solved on the finest grid. The grid density outside this interfacial region is determined using a hybrid scheme based on a local error estimator and rules that ensure smooth grading of the mesh. Please see the original papers for the details about the numerical techniques. ${ }^{[21]}$

Echebarria et al. ${ }^{[15]}$ showed that mesh-converged results can be obtained with $W_{0} / d_{0}$ as large as 50 . With the material properties given in Table I, we have $d_{0} \approx$ $1 \times 10^{-8} \mathrm{~m}$. The smallest expected tip radius is around $1 \times 10^{-6} \mathrm{~m}$, and using the rule that the tip radius should be at least ten times the interface width, ${ }^{[15]}$ we have $R_{\mathrm{tip}} / W_{0}=10, W_{0}=1 \times 10^{-7} \mathrm{~m}$, and thus $W_{0} / d_{0} \approx 10$. We then obtain $\tilde{D}_{\ell}=5.5$, which implies that $\tau_{0}=4.6 \times$ $10^{-5} \mathrm{~s}$. All other physical values, such as the thermal length, the pulling velocity, and the simulation box size are then non-dimensionalized with these values of $W_{0}$ 
Table I. Material Properties Chosen for the Phase-Field

Simulations and Values Found in the Literature for the Composition and Temperature Considered

\begin{tabular}{llll}
\hline Property & \multicolumn{1}{c}{ Value } & \multicolumn{1}{c}{ Units } & \multicolumn{1}{c}{ Ref. } \\
\hline$C_{\infty}$ & 0.1 & mass frac Zn & - \\
$m_{\ell}$ & -170 & $\mathrm{~K}$ & {$[17]$} \\
$k_{0}$ & 0.45 & - & {$[17]$} \\
$T_{\mathrm{M}}$ & $933(660)$ & $\mathrm{K}\left({ }^{\circ} \mathrm{C}\right)$ & {$[17]$} \\
$D_{\ell}$ & $1.2 \times 10^{-9}$ & $\mathrm{~m}^{2} / \mathrm{s}$ & {$[16]$} \\
$\Gamma_{s \ell}$ & $1.05 \times 10^{-7}$ & $\mathrm{Km}$ & {$[18,19]$} \\
$\rho L_{\mathrm{f}}$ & $1 \times 10^{9}$ & $\mathrm{~J} / \mathrm{m}^{3}$ & {$[19]$} \\
$\rho c_{\mathrm{p}}$ & $3 \times 10^{6}$ & $\mathrm{~J} / \mathrm{m}^{3} \mathrm{~K}$ & {$[20]$} \\
\hline
\end{tabular}

Note that the value of $D_{\ell}$ has been increased by a factor of two in the simulations, compared to the literature value in Ref. [16].

and $\tau_{0}$. In the calculations presented below, we use $d x_{\min }=0.75 W_{0}$ and a time step in the explicit solution scheme of $0.05 \tau_{0}$.

Since our study is focused on the role of anisotropy, it is essential that we measure and correct for grid anisotropy. All of the finite elements are cubes, whose edge length scales in powers of two with refinement level. We therefore assume that the grid anisotropy can be written in the same form as Eq. [5], truncated after the first anisotropic term, i.e., $a_{1}^{\text {grid }} \neq 0, a_{2}^{\text {grid }}=0$. To determine $a_{1}^{\text {grid }}$, we grew equiaxed dendrites whose crystallographic axes were rotated by various angles with respect to the Cartesian grid. We then measured the difference in growth speed in the different directions and adjusted the grid anisotropy in order to eliminate the differences. The amount of grid anisotropy depends on the minimum grid spacing and was fixed in the present case to $a_{1}^{\text {grid }}=-0.019$. Because the grid coarsening is controlled by an error estimator, the grid anisotropy is not affected by the grid outside the interfacial region. We note that one cannot completely eliminate the grid anisotropy in this way, as second-order effects $\left(a_{2}^{\text {grid }} \neq 0\right)$ may still be present. We estimate that the residual grid anisotropy corresponds to $a_{1} \approx 0.001$. This will affect the simulations by providing a source of noise to induce side branching, but should not affect the fundamental orientation selection at the dendrite tip.

\section{RESULTS}

\section{A. Equiaxed Growth}

Our first objective was to determine whether the results obtained by Haxhimali et $a l .{ }^{[1]}$ for pure materials held in simulations for binary materials, where $D_{s} \ll D_{\ell}$. To that end, we performed a survey of anisotropy parameter space for equiaxed growth from a small spherical seed in an isothermal melt at fixed supersaturation $\Omega$, defined as

$$
\Omega=\frac{C_{\ell}^{0}-C_{\infty}}{C_{\ell}^{0}\left(1-k_{0}\right)}
$$

where $C_{\ell}^{0}=\left(T_{0}-T_{\mathrm{f}}\right) / m_{\ell}$ and $T_{0}$ defines the temperature for the isothermal solidification. Each simulation was carried out in one octant of a cubic domain, with all faces having zero-flux boundary conditions. The $\langle 100\rangle$ crystallographic axes were either aligned with the Cartesian directions of the grid or rotated by $45 \mathrm{deg}$ about the [100] to verify the grid anisotropy correction. An initial one-eighth spherical seed of radius $R_{0}=5-10 \times W_{0}$ was placed at one corner of the computational domain.

The far-field supersaturation, defined as an input parameter, was either $\Omega=0.4$ or $\Omega=0.25$ in the results presented below. The initial concentration field was then defined as

$$
C= \begin{cases}k_{0} C_{\ell}^{0} & r \leq R_{0} \\ C_{\ell}^{0}\left[1+\Omega\left(1-k_{0}\right)\left(R_{0} / r-1\right]\right) & r>R_{0}\end{cases}
$$

The computational domain in units of $W_{0}$ was $768^{3}$ with $d \tilde{x}_{\min }=0.75$. In a uniform grid, this would require $4.5 \times 10^{8}$ grid points, whereas in our simulations, the grid typically started with about 5000 nodes and at the end of the simulations, there were $1-4 \times 10^{6}$ nodes. The calculations were run on both 2.93 and $3.30 \mathrm{GHz}$ Intel Xeon CPUs. Run times to generate fully developed microstructures at $\Omega=0.4$ (12000 time steps) were typically less than one day. The execution time is related to the complexity of the microstructure that forms because the size of the adaptive grid scales with the amount of solid-liquid interfacial area.

In the investigations by Haxhimali et al. ${ }^{[1,27]}$, line scans were performed by varying $a_{1}$ at several fixed values of $a_{2}(-0.005,-0.01,-0.02)$. On each scan, they found that low enough values of $a_{1}$ resulted in $\langle 110\rangle$ dendrites, and at high values of $a_{1},\langle 100\rangle$ dendrites appeared. In the intermediate region, mixed structures were obtained, which they termed "hyperbranched." We surveyed the anisotropy parameter space using a similar approach, i.e., using line scans. Since all of the available experimental and MD data ${ }^{[28]}$ show that these parameters are in the range $0 \leq a_{1} \leq 0.12$ and $-0.01 \leq a_{2} \leq 0$, the $\left(a_{1}, a_{2}\right)$ couples were chosen as indicated in Figure 2, which also provides a sampling of the computed microstructures. In particular, the diagonal scan line was chosen to go approximately through the following points: $\left(a_{1},-a_{2}\right)=(0.07,0.0)$, measured by Napolitano et al. ${ }^{[29]}$ for Al-Si alloys; $\left(a_{1},-a_{2}\right)=(0.05,0.0045)$ obtained by MD simulations using the embedded atom method $^{[30]}$; and $\left(a_{1},-a_{2}\right)=(-0.025 \pm 0.023,0.024 \pm$ $0.014)$ measured recently by Friedli for quenched droplets in Al-82 wt pct Zn alloys. ${ }^{[12]}$

We found results similar to those reported by Haxhimali et al. ${ }^{[1,27]}$ with respect to the transitions among the three types of microstructures. Looking first at the line scan $a_{2}=-0.01$, we see in Figure 2 that $\langle 110\rangle$ dendrites form when $a_{1}=0$. Notice that the tip has an elliptical rather than circular shape in a transverse section, reflecting the underlying symmetry of the $\langle 110\rangle$ direction. As $a_{1}$ increases, the strength of the anisotropy along $\langle 100\rangle$ increases. At first, the $\langle 110\rangle$ tips become broader and more elliptical in (100) planes, and they grow more slowly. As $a_{1}$ increases further, the tips become sufficiently flat that they split along the $\langle 110\rangle$ axes (see the case $a_{1}=0.04, a_{2}=-0.01$ in Figure 2). 
These tips continue to grow and eventually produce hyperbranched structures. Increasing $a_{1}$ still more in this line scan, the $\langle 110\rangle$ tips split earlier and thus grow larger (cf. $\left.\left(a_{1}, a_{2}\right)=(0.06,-0.01)\right)$. As one approaches the boundary between the hyperbranched and $\langle 100\rangle$ dendrites, the dendrites present four tips split along the $\langle 100\rangle$ axes, which then merge into single tips oriented along the $\langle 100\rangle$ for sufficiently large $a_{1}$.

We note that although the tip growth velocity has converged for the extreme cases with clear $\langle 100\rangle$ and $\langle 110\rangle$ dendrites, the speeds of the double and quadruple tips are still in their initial transient at the end of the simulation time considered here. Thus, our simulations should be performed for longer times to verify that those structures persist. However, our main objective in this part of the study has been achieved, i.e., we have verified that the results obtained by Haxhimali et al. for a pure material are also observed in binary alloys.

Figure 2 also includes the two lines corresponding to our simplified principles for selection (see Figure 1). Both of the lines, i.e., maximum surface energy $\left(a_{1}=-3 a_{2}\right)$ or minimum stiffness trace $\left(a_{1}=-20 / 3 a_{2}\right)$, could be interpreted as predicting hyperbranched dendrites for intermediate values of $\left(a_{1}, a_{2}\right)$, but the transition is clearly much broader than either line.

Although the locations of the boundaries between the different morphologies are remarkably similar to those reported by Haxhimali et al. for thermal dendrites, the details of the dendritic structures in the hyperbranched region are somewhat different. In particular, Haxhimali et al. observed less branching in the off-axis directions. We attribute this difference to the fact that the simulations presented here correspond to solutal dendrites growing at relatively large supersaturation $(\Omega=0.4)$, whereas Haxhimali et al. considered a pure material at very low undercooling, $\Delta=0.05$. The higher supersaturation produces higher growth velocities, which in turn introduce more noise into the simulation, leading to more extensive side branching. To examine the role of $\Omega$ on the morphology of the equiaxed crystal, we performed a few simulations at $\Omega=0.25$. Figure 3 compares equiaxed dendrites computed for the parameter set $a_{1}=0.08, a_{2}=-0.01$ with $\Omega=0.4$ and $\Omega=0.25$. Although the envelopes of the two dendrites are similar, the amount of "activity" in the $\langle 110\rangle$ directions is significantly less at lower supersaturation. We note that

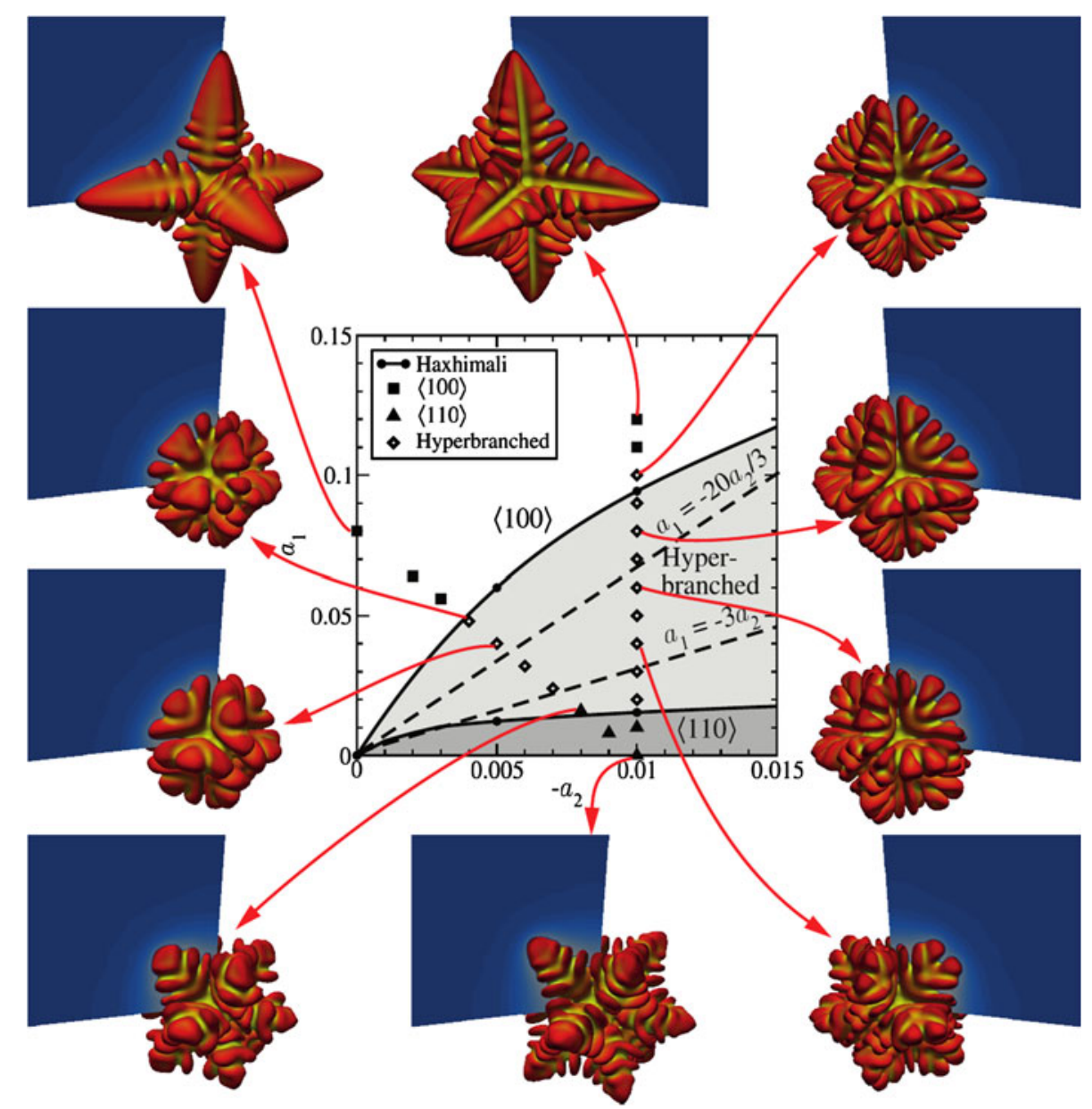

Fig. 2-Phase-field results for equiaxed growth of Al-0.1 $\mathrm{Zn}$ alloy with varying anisotropy parameters, and $\Omega=0.4$. The scale and orientation of all dendrites are identical. The solid lines in the center graph represent the limits between the domains $\langle 100\rangle$, hyperbranched and $\langle 110\rangle$ structures, reported by Haxhimali et al. ${ }^{[1]}$ The present calculations show very similar results for the locations of the boundaries. The two dashed lines correspond to the simple selection criteria (maximum surface energy and minimum stiffness) shown in Fig. 1. The dendrite surface is colored by concentration, and the grayscale background shows the liquid concentration field in the plane of the dendrite tip. 


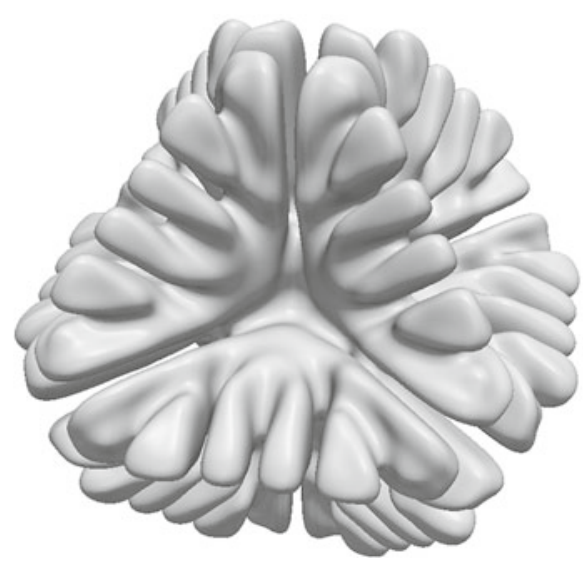

(a) $\Omega=0.4, t=14000$

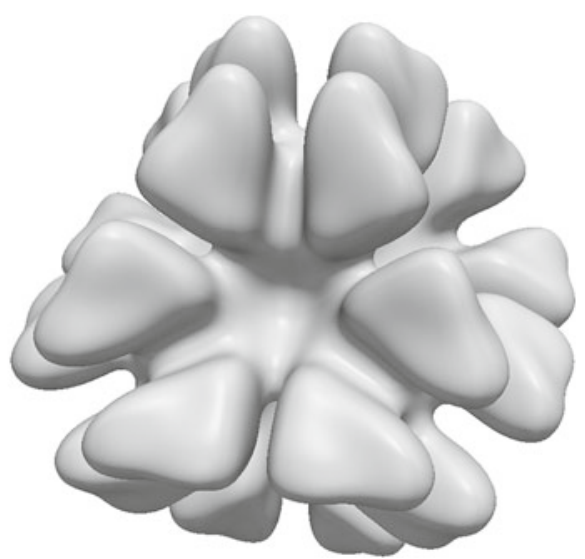

(b) $\Omega=0.25, t=45000$

Fig. 3 - Comparison of computed equiaxed dendrites with $a_{1}=0.08, a_{2}=-0.01$, at two different supersaturations $\Omega$. The times were chosen from the two simulations such that the dendrite tips were at approximately the same distance from the center.

the computations become increasingly expensive as the supersaturation decreases because the dendrites grow much more slowly, and thus the simulations must be run for a longer time and to larger overall size.

\section{B. Directional Solidification}

In order to directly compare the simulations with the experiments in the companion paper, we performed a similar exploration of anisotropy parameter space for DS. For these simulations, we investigated anisotropy parameters along the diagonal line in Figure 5 running from $\left(a_{1}, a_{2}\right)=(0.08,0)$ to $(0,-0.01)$, using the domain shown schematically in Figure 4.

There is an additional variable introduced in DS compared to equiaxed growth, which is the orientation of the crystallographic directions with respect the thermal gradient, parallel to the pulling velocity. In the experiment, there are many viable nuclei at the beginning of solidification and one anticipates that the most favorably oriented crystals will dominate after a prolonged period of growth (cf. Reference 31, Section 11.4). We identify these orientations in the simulations as those that have grown the farthest in the pulling direction after 27000 time steps, a period long enough to clearly establish the structure. This also corresponds to the structure with the lowest tip undercooling and the fastest growing orientation.

We refer to the Cartesian axes of the computational domain as $(\hat{\boldsymbol{x}}, \hat{\boldsymbol{y}}, \hat{\boldsymbol{z}})$, with $\hat{\boldsymbol{x}}$ parallel to the pulling direction and the imposed temperature gradient $G$. The initial quarter-sphere seed was placed as illustrated in Figure 4, centered on the edge of the simulation domain defined by the line $x=z=0$. The vertical faces with normal vector $\pm \hat{\boldsymbol{z}}$ were treated as symmetry planes, and those with normal vector $\pm \hat{\boldsymbol{y}}$ were made periodic. In the experiments, we saw that the primary dendrite trunks were bisected by a vertical (001) plane. In view of this, we oriented the crystal such that the vertical $x-y$ plane corresponded to the (001) plane. Thus, we have $\hat{z} \|[001]$, as indicated in Figure 4. In this way, various orientations could be obtained by rotating

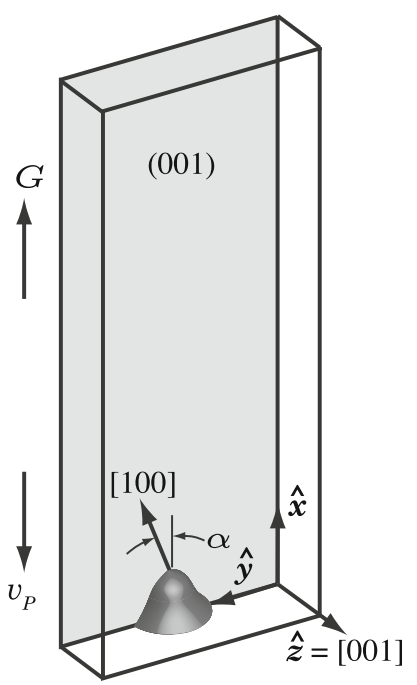

Fig. 4-DS simulation domain and seed position configuration. The front and back faces $(\vec{n}= \pm \hat{z})$ are symmetry planes, parallel to the (001) plane of the crystal, and the side faces $(\vec{n}= \pm \hat{\boldsymbol{y}})$ are periodic. The crystal axes are rotated with respect to $\hat{\boldsymbol{x}}$ by an angle $\alpha$ that is specified in the simulation.

the crystal axes around the $z$-axis, as measured by the Euler angle $\alpha$ between [100] and $\hat{\boldsymbol{x}}$. We performed a scan in $\alpha$ from zero to $45 \mathrm{deg}$ in increments of $5 \mathrm{deg}$ for each anisotropy parameter pair.

The horizontal faces at the top and bottom of the computational domain also had zero normal derivative boundary conditions. The pulling velocity is normal to these faces, so the material is advected into the domain at the top and leaves at the bottom. The upper surface was always far enough from the crystal as to be unaffected by the solute diffusion field ahead of the solid.

In DS, we use the supersaturation parameter $\Omega_{0}$, defined in Eq. [17], to set the approximate solid fraction at the base of the domain, $f_{m}$, using the lever rule. Note that the actual solid fraction will be less than $f_{m}$ computed in this way, due to solute redistribution 


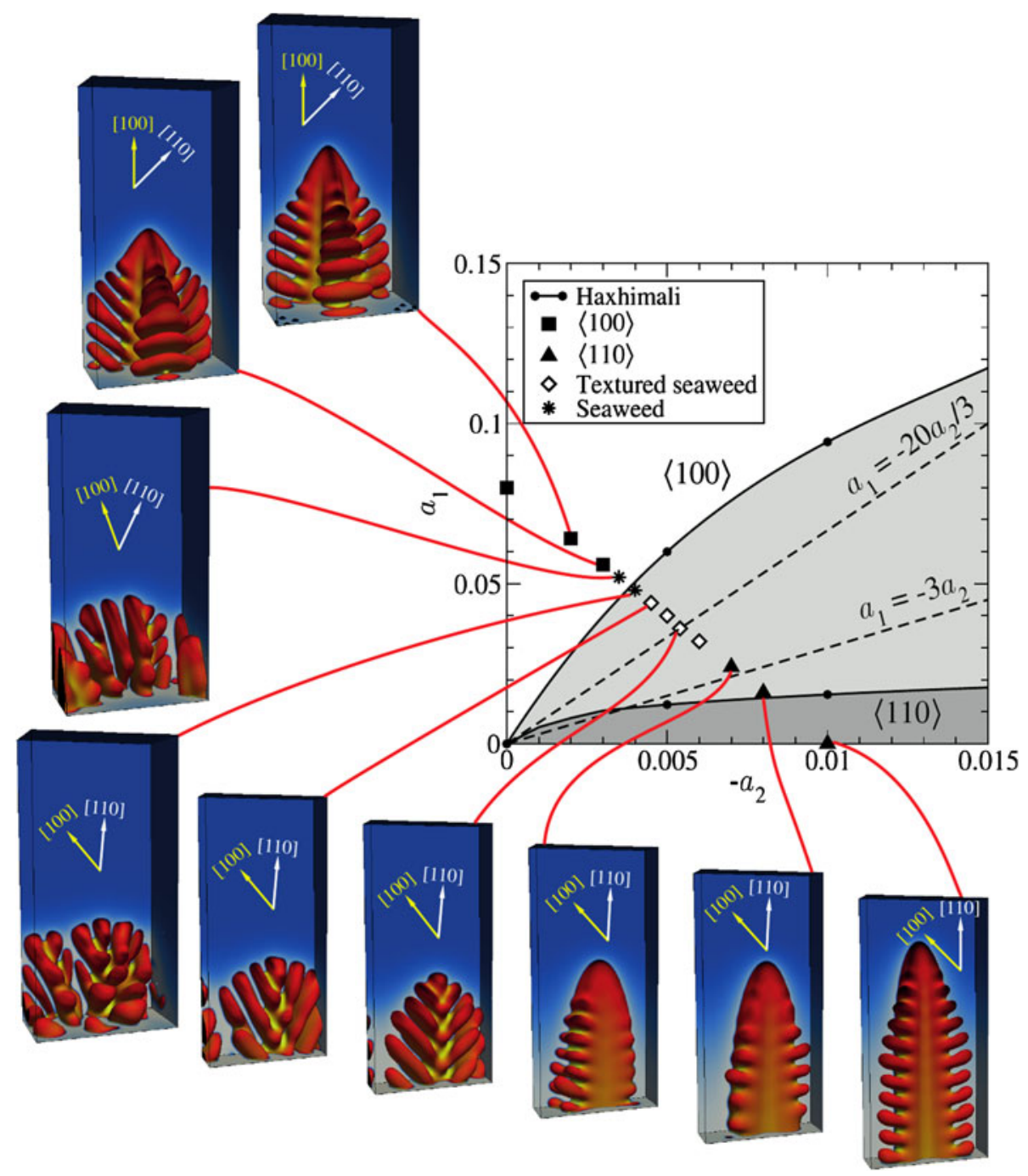

Fig. 5-Computed microstructures for the orientation with the lowest undercooling for various values of anisotropy parameters along the line indicated. For each selected microstructure, the orientation of [100] and [110] is shown. The thermal gradient and pulling direction are aligned with the vertical axis of the domain. The dendrite surface is colored by concentration, and the grayscale background shows the liquid concentration field in the plane of the dendrite tip.

effects, but we do not require an exact value for our purposes.

$$
f_{m}\left(\Omega_{0}\right)=\frac{k_{0} \Omega_{0}}{1-\left(1-k_{0}\right) \Omega_{0}}
$$

If $\Omega_{0}=1$, the entire mushy zone is present in the simulation domain, which would require the domain to be very large to accommodate the entire dendrite, leading to long computation times. We typically set $\Omega_{0}=0.45$, which for $k_{0}=0.45$ corresponds to $f_{m}=0.269$. The temperature at the base of the domain can be computed from the phase diagram, $T_{0}=T_{\text {liq }}-\left(T_{\text {liq }}-T_{\text {sol }}\right) f_{m}$.

When the primary dendrite trunk is oriented nearly along [100], the domain should have an aspect ratio in the horizontal plane of approximately 2:1 to accommodate the side branches. On the other hand, when the primary dendrite trunk is close to [110], the aspect ratio should be 7:2 to accommodate the $\langle 110\rangle$ side branches. We used two different computational domains:
$384 \times 192 \times 96$ for the [100] orientations and $360 \times$ $168 \times 48$ for the [110] orientations. One could tell early on in the calculations which direction was being selected, and we made sure to use the appropriate domain for the structure that appeared. In the case of seaweed structures, both domains were examined. Note that the size of the domain in the $y-z$ plane was chosen to correspond approximately to the observed primary spacing and was not varied in the simulations.

The simulation parameters common to all of the DS cases are listed in Table II. The physical parameters used in these simulations were the same as those used in the equiaxed growth simulations, given in Table I. We started the calculations with the same radius quarter sphere as in the equiaxed case, ran the simulation for 5000 time steps in a fixed frame $\left(\tilde{v}_{\mathrm{F}}=0\right)$ to establish the seed, then restarted the calculation with $\tilde{v}_{\mathrm{F}}=\tilde{v}_{\mathrm{P}}$, and continued the run to 27000 total time steps. This typically resulted in well-defined dendrites with $7.5 \times$ 
Table II. Directional Solidification Simulation Parameters and Their Dimensional Equivalents

\begin{tabular}{lll}
\hline $\begin{array}{l}\text { Dimensionless } \\
\text { Parameter }\end{array}$ & Value & \multicolumn{1}{c}{$\begin{array}{c}\text { Dimensional } \\
\text { Equivalent }\end{array}$} \\
\hline$\Delta x / W_{0}$ & 0.75 & $\Delta x=7.5 \times 10^{-8} \mathrm{~m}$ \\
$a_{1}^{\text {grid }}$ & -0.019 & $\mathrm{~N} / \mathrm{A}$ \\
$\Delta t / \tau_{0}$ & 0.05 & $\Delta t=2.3 \times 10^{-6} \mathrm{~s}$ \\
$\tilde{l}_{\mathrm{T}}$ & $1 \times 10^{4}$ & $G=20777 \mathrm{~K} / \mathrm{m}$ \\
$\tilde{v}_{\mathrm{P}}$ & 0.25 & $v_{\mathrm{P}}=543 \mu \mathrm{m} / \mathrm{s}$ \\
$\tilde{v}_{\mathrm{F}}$ & 0.25 & $v_{\mathrm{F}}=543 \mu \mathrm{m} / \mathrm{s}$ \\
$\Omega$ & 0.45 & $\mathrm{~N} / \mathrm{A}$ \\
\hline
\end{tabular}

$10^{5}$ to $2.5 \times 10^{6}$ nodes, and ran in about 1 to 3 days on the same machines mentioned earlier. Once again, the execution time depended on the complexity of the microstructure.

The results for the fastest growing, lowest tip undercooling microstructures are summarized in Figure 5. For convenience of reference, we have included from Figure 2 the dividing lines associated with the surface energy and stiffness trace criteria, and the shaded regions where $\langle 100\rangle,\langle 110\rangle$ and hyperbranched structures were found in equiaxed solidification. Please note that all of these simulations were run for the same amount of time. Therefore, smaller solid structures are reflective of slower growth and higher undercooling, compared to the larger ones. At the extreme ends of the scan line, we see the expected $\langle 100\rangle$ and $\langle 110\rangle$ primary dendrites, and the most interesting structures appear in the intermediate region.

Starting from $\left(a_{1},-a_{2}\right)=(0,0.01)$, where we have $\langle 110\rangle$ dendrites, and progressing along our scan line, Figure 5 shows that the $\langle 110\rangle$ dendrite tip becomes increasingly blunt. Notice that when the dendrite is oriented with [110] inclined by $3-5$ deg with respect to the pulling directions, side branches are emitted preferentially on the side facing the melt ahead of the growing tip. The primary trunk becomes less and less distinct as we approach the line separating the regions of $\langle 110\rangle /\langle 100\rangle$ minimum stiffness, $a_{1}=-20 a_{2} / 3$. After that line is crossed, (see Figure $5 \quad\left(a_{1}, a_{2}\right)=$ $(0.044,-0.0045))$, the tip and main trunk disappear, and we have only advancing side branches that curve outward as they grow. The lowest undercooling orientation also has rotated such that the [110] direction is 5 deg away from the pulling direction. This orientation corresponds to $[230] \| \hat{\boldsymbol{x}}$, which helps to explain the $\langle 320\rangle$ texture reported by Gonzales et al. ${ }^{[4]}$ for $C_{\infty}=50$ wt pet $\mathrm{Zn}$. Moving further along the line scan, the selected orientation rotates continuously toward $[100] \| \hat{\boldsymbol{x}}$, and textured seaweeds appear close to and on both sides of the boundary where the $\langle 100\rangle$ dendrites appeared in the simulations of equiaxed growth. Finally, $\langle 100\rangle$ dendrites appear. Thus, for DS, the minimum stiffness criterion provides an approximate predictor for the onset of seaweed structures.

The growth mechanism for the textured seaweeds can be better understood with the aid of Figure 6, which shows the intersection of the surface $\psi=0$ with the (001) plane $(z=0)$. The figure shows a temporal



Fig. 6-Temporal sequence showing the advancement of the dendrite tip on the (001) plane for the case $\left(a_{1}, a_{2}\right)=(0.048,-0.004)$. Notice that the tip is broad, and oscillates from side to side, emitting side branches at different heights with respect to the pulling direction.

sequence of the dendrite tip position for the case $\left(a_{1}, a_{2}\right)=(0.048,-0.004)$ between 19000 and 31000 time steps, in increments of 1000 . The contours are shown transformed back to the reference frame fixed in space, rather than the computational domain traveling with the pulling velocity. One can see clearly that the tip is very broad and oscillates from side to side. As it does so, it emits side branches at different heights with respect to the pulling axis, corresponding to the location of the tip. This phenomenon occurs because both anisotropy components are relatively weak, and for this case, their effects are also nearly equal in magnitude. This is the same mechanism that was found by Akamatsu et al. ${ }^{[32]}$ to produce seaweeds in 2-D thin samples.

\section{DISCUSSION}

The simulations of equiaxed and DS demonstrate that as the surface energy anisotropy varies over a fairly narrow range, there is a continuous transition in dendrite trunk orientation between $\langle 100\rangle$ and $\langle 110\rangle$. The intermediate region between these two extremes is characterized by hyperbranched structures in equiaxed growth. The additional constraint imposed by the 
thermal gradient leads instead to textured seaweed structures in directional solidification.

One of our objectives in this study was to correlate the anisotropy parameters with $\mathrm{Zn}$ content. We can do so only qualitatively for several reasons. We do not really know whether the line we chose to scan along actually corresponds precisely to the alloys in the Al-Zn system. Recall that this line was chosen based on published values from MD studies of Al surface energy anisotropy for low $\mathrm{Zn}$ content and a measured value for high $\mathrm{Zn}$ alloys obtained experimentally. All of these values are subject to a fairly large degree of uncertainty. If we nevertheless assume that our line scan corresponds to $\mathrm{Zn}$ content, then we can attempt to correlate our calculation results with the experimental findings of Gonzales et al. ${ }^{[3]}$

The result is shown in Figure 7 . The computational results are presented as a contour plot, showing the height of the computed dendritic structure after 27000 time steps for all of the anisotropy pairs and orientations considered, indicated by open circles. (See the scale at the top of the figure.) We also highlight by a red line the orientations with the largest growth and minimum undercooling for each anisotropy pair along the line. Superimposed on these data, we show as green squares the experimentally measured orientations reported by Gonzales et al. ${ }^{[3]}$ Based on the idea that at. pct is a more representative measure of how $\mathrm{Zn}$ in the solid modifies the solid-liquid interfacial energy, those data, originally reported as wt pet $\mathrm{Zn}$, have been rescaled to at. pct $\mathrm{Zn}$ at the corresponding solidus $k_{0}^{m} X_{\infty}$, where $k_{0}^{m}$ is the segregation coefficient when the composition $X_{\infty}$ is measured in at. pct. (See the scale at the bottom of the figure.) The relevant portion of the equilibrium phase diagram is also shown for reference. The results presented in Figure 7 can be used to correlate the anisotropy parameters with the composition by comparing the scales on the top and bottom of the graph. The result is given by

$$
\begin{aligned}
a_{1} & =0.064-1.33 \times 10^{-3}\left(k_{0}^{m} X_{0}\right) ; \\
-a_{2} & =0.002+1.67 \times 10^{-4}\left(k_{0}^{m} X_{0}\right)
\end{aligned}
$$

The agreement between the experimental and computational results seems remarkable, but we must also be clear that this is somewhat artificial. The horizontal axis of the experimental data $\left(k_{0}^{m} X_{0}\right)$ was translated and stretched by a constant factor to make the beginning and end of DOT agree with the calculations. If we had chosen a different, but similar, line scan in anisotropy space, still crossing the three regions from $\langle 110\rangle$ to $\langle 100\rangle$, the result would have been similar, although the scaling might be different. Nevertheless, we obtain for anisotropy of pure $\mathrm{Al}\left(a_{1}, a_{2}\right)=(0.064,-0.002)$, which is quite close to the measurement of Napolitano et al. ${ }^{[29]}$ in dilute Al-Si alloys. We should also note that instead of the solid composition at the liquidus temperature $\left(k_{0}^{m} X_{\infty}\right)$, one should also correct for the fact that the liquid at the tip is actually at a higher $\mathrm{Zn}$ content. For all of the selected microstructures in Figure 7, the supersaturation measured from the concentration ahead of the dendrite tip was $\Omega_{\text {tip }} \approx 0.3 \pm 0.05$. For the nominal composition $C_{\infty}=0.1$, this amounts to a tip undercooling of about $3.5 \mathrm{~K}$. The correction is thus quite small, and in any case within the margin of error of our choice of the line scan in anisotropy space. We also note that the simulation domain represents a very small fraction of the mushy zone, and it is therefore reasonable to treat the anisotropy parameters as constant over the entire region.



Fig. 7-Contour map showing the maximum height of the computed dendrite for each anisotropy couple $\left(a_{1}, a_{2}\right)$, at various orientations of the [100] direction with respect to the pulling direction in the (001) plane. The red circles indicate, for each couple $\left(a_{1}, a_{2}\right)$, the angle corresponding to the most developed (lowest undercooling) structure. We also superimpose as green squares the experimental results reported by Gonzales et $a l^{[3]} \mathrm{A}$ portion of the $\mathrm{Al}-\mathrm{Zn}$ phase diagram is shown in gray (Color figure online). 


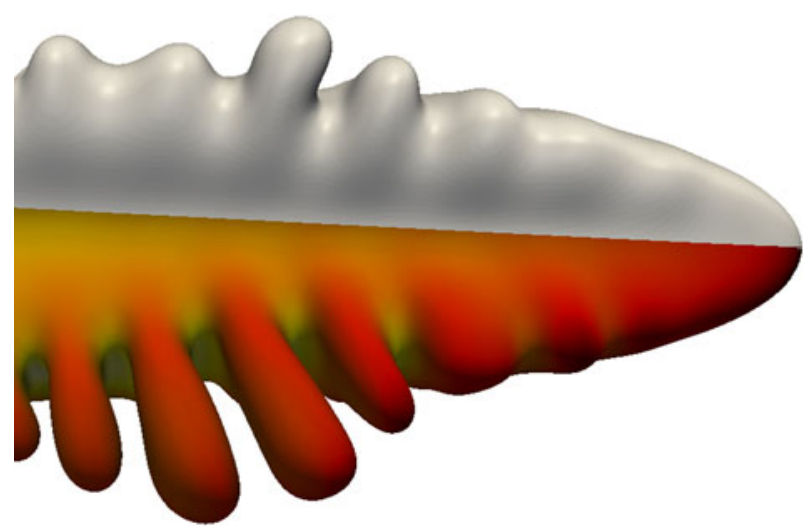

Fig. 8-Comparison of dendrites computed in two different size domains for $a_{1}=0.016, a_{2}=-0.008, \alpha=42^{\circ}$. The dendrite computed in the smaller domain is above and the one computed in the larger domain below. The tips are virtually identical, but the larger domain admits larger side branches.

There are some additional limitations to the simulations that should be noted. We did not add noise to the equations, and hence we can draw only limited conclusions about side branch formation. The residual grid anisotropy is thus the main remaining source of noise in the simulations. However, since the dendrite surface is itself morphologically unstable, the grid noise is still sufficient to induce side branching. In some cases, the boundaries of the simulation domain affect the formation of the side branches at some distance from the tip. This is an indication that the simulation domain is too small to accommodate the structure. An example of this phenomenon occurs for the anisotropy parameters $\left(a_{1},-a_{2}\right)=(0.016,0.008)$. We ran a simulation in a 30 pet larger domain, $480 \times 216 \times 60$, keeping all other parameters unchanged, and the computed dendrites are compared side-by-side in Figure 8. The dendrite computed in the smaller domain is at the top. One can see that the tips are nearly identical, and that the side branches are indeed more developed when the constraint of the lateral boundaries is moved back. The fact that the tip shapes are the same, however, indicates that our study, which focuses on the dynamics of tip selection, is not strongly affected by the domain size.

\section{CONCLUSIONS}

We performed a systematic exploration of anisotropy parameter space to examine the hypothesis that the DOT in DS Al-Zn alloys can be explained as being due to a continuous transition in anisotropy of the solidliquid interfacial energy with $\mathrm{Zn}$ content. Our results show a transition similar to that observed in experiments, from $\langle 100\rangle$ dendrites, to textured seaweeds, and then to $\langle 110\rangle$ as the anisotropy is varied.

In particular, for DS in the intermediate region of parameter space separating the regions where $\langle 100\rangle$ and $\langle 110\rangle$ form, we find a transition over a narrow range of anisotropy parameters where the orientation rotates continuously from $\langle 110\rangle$ to $\langle 100\rangle$ parallel to the thermal gradient and pulling direction. By comparing these results with the experimental results of Gonzales and Rappaz, ${ }^{[3,4]}$ we are able to correlate the anisotropy parameters with the corresponding narrow range of solid compositions, expressed in at. pct.

\section{ACKNOWLEDGMENTS}

The authors gratefully acknowledge the support of the Swiss National Fund (Grant Nos. 200020-113260 and 200020-121598), the European Community's Seventh Framework Program under grant agreement No. 229108 (MIntWeld), and the US National Aeronautics and Space Administration (NASA) under grant NNX10AJ73G.

\section{REFERENCES}

1. T. Haxhimali, A. Karma, F. Gonzales, and M. Rappaz: Nat. Mater., 2006, vol. 5, pp. 660-64.

2. J. Friedli, J. Fife, P. Di Napoli, and M. Rappaz: Metall. Mater. Trans. A, 2013. DOI: 10.1007/s11661-013-1912-7.

3. F. Gonzales and M. Rappaz: Metall. Mater. Trans. A, 2006, vol. 37A, pp. 2797-806.

4. F. Gonzales and M. Rappaz: Metall. Mater. Trans. A, 2008, vol. 39A, pp. 2148-60.

5. W.W. Mullins and R.F. Sekerka: J. Appl. Phys., 1963, vol. 34, pp. $323-29$.

6. S.K. Chan, H.H. Reimer, and M. Kahlweit: J. Cryst. Growth., 1976, vol. 32, pp. 303-15.

7. W.R. Fehlner and S.H. Vosko: Can. J. Phys., 1976, vol. 54, pp. 2159-69.

8. R.E. Napolitano and S. Liu: Phys. Rev. B, 2004, vol. 70, pp. 1-11.

9. A. Karma and W.J. Rappel: Phys. Rev. E, 1998, vol. 57, pp. 432349.

10. C. Herring: in The Physics of Powder Metallurgy, W. Kingston, ed., McGraw-Hill, New York, 1951, pp. 143-77.

11. D. Du, H. Zhang, and D. Srolovitz: Acta Mater., 2007, vol. 55, pp. $467-71$.

12. J. Friedli: Ph.D. Thesis, EPFL, 2011.

13. D. Hoffman and J. Cahn: Surf. Sci., 1972, vol. 31, pp. 368-88.

14. A. Karma: Phys. Rev. Lett., 2001, vol. 87, pp. 045501-1-045501-4.

15. B. Echebarria, R. Folch, A. Karma, and M. Plapp: Phys. Rev. E, 2004, vol. 70, pp. 061604-1-061604-22.

16. Y. Du, Y. Chang, B. Huang, W. Gong, Z. Jin, H. Xu, Z. Yuan, Y. Liu, Y. He, and F.Y. Xie: Mater. Sci. Eng. A Struct., 2003, vol. 363 , pp. $140-51$.

17. S. Mey and G. Effenberg: Z. Metallkd., 1986, vol. 77, pp. 449-53.

18. J. Morris, M. Mendelev, and D. Srolovitz: J. Non-Cryst. Solids, 2007, vol. 353, pp. 3565-69.

19. J. Valencia and P. Quested: in ASM Handbook, vol. 22B, D. Furrer and S. Semiatin, eds., ASM International, Materials Park, OH, 2010, pp. 18-32.

20. C. Smithells and E. Brandes: in Smithells Metals Reference Book, 6th ed., E. Brandes, ed., Butterworths, London, 1983, p. 14-1.

21. J.H. Jeong, N. Goldenfeld, and J. Dantzig: Phys. Rev. E, 2001, vol. 64, pp. 041602-1-041602-14.

22. J.H. Jeong, J. Dantzig, and N. Goldenfeld: Metall. Mater. Trans. $A$, 2003, vol. 34 A, pp. 459-66.

23. E. Burman, A. Jacot, and M. Picasso: J. Comput. Phys., 2004, vol. 195, pp. 153-74.

24. N. Provatas, M. Greenwood, B. Athreya, N. Goldenfeld, and J. Dantzig: Int. J. Mod. Phys. B, 2005, vol. 19, pp. 4525-65.

25. B. Athreya, J. Dantzig, S. Liu, and R. Trivedi: Philos. Mag., 2006, vol. 86 , pp. $3739-56$.

26. J. Narski and M. Picasso: Comput. Methods Appl. Mech. Eng., 2007, vol. 196, pp. 3562-76.

27. T. Haxhimali: Ph.D. Thesis, Northeastern University, 2006. 
28. M. Asta, C. Beckermann, A. Karma, W. Kurz, R. Napolitano, M. Plapp, G. Purdy, M. Rappaz, and R. Trivedi: Acta Mater., 2009, vol. 57, pp. 941-71.

29. R.E. Napolitano, S. Liu, and R. Trivedi: Interface Sci., 2002, vol. 10, pp. 217-32.
30. F. Ercolessi and J. Adams: Europhys. Lett., 1994, vol. 26, pp. 583-88.

31. J. Dantzig and M. Rappaz: Solidification, 1st ed., EPFL Press, Lausanne, 2009.

32. S. Akamatsu, G. Faivre, and T. Ihle: Phys. Rev. E, 1995, vol. 51, pp. 4751-80. 